\title{
Qilisheng Immunoregulatory Oral Solution
}

National Cancer Institute

\section{Source}

National Cancer Institute. Qilisheng Immunoregulatory Oral Solution. NCI Thesaurus.

Code C155882.

An oral solution containing Spondias axillaris, Panax ginseng, schisandra berry, hawthorn, soybean and an as of yet not elucidated bacterium, with potential immunomodulating activity. Upon oral administration of the qilisheng immunoregulatory oral solution, the ing redients in qilisheng may modulate the immune system. 